\title{
A Flexible Approach to Inference in Semiparametric Regression Models with Correlated Errors Using Gaussian Processes
}

\author{
Heping $\mathrm{He}$ \\ College of Finance and Statistics, Hunan University (North Campus), Changsha, Hunan 410082, \\ P.R. China \\ Thomas A. Severini \\ Department of Statistics, Northwestern University, 2006 Sheridan Road, Evanston, IL 60208, \\ USA
}

\begin{abstract}
Consider a semiparametric regression model in which the mean function depends on a finite-dimensional regression parameter as the parameter of interest and an unknown function as a nuisance parameter. A method of inference in such models is proposed, using a type of integrated likelihood in which the unknown function is eliminated by averaging with respect to a given distribution, which we take to be a Gaussian process with a covariance function chosen to reflect the assumptions about the function. This approach is easily implemented and can be applied to a wide range of models using the same basic methodology. The consistency and asymptotic normality of the estimator of the parameter of interest are established under mild conditions. The proposed method is illustrated on several examples.

Keywords: Semiparametric model, Gaussian process regression, Generalized least squares, Restricted maximum likelihood.
\end{abstract}

\section{Introduction}

Consider semiparametric regression models in which the mean of the response depends on a finite-dimensional regression parameter $\beta$ as the parameter of inter-

Email addresses: heping . he@out look . com (Heping He), severini@northwestern.edu (Thomas A. Severini) 
est as well as an unknown function $\gamma(\cdot)$ as a nuisance parameter; in addition, such models generally contain a parameter $\phi$ parameterizing the covariance structure of the errors in the model. Our goal is non-Bayesian inference for the parameter $\beta$ in the presence of the unknown function $\gamma$.

In the usual approaches to inference in semiparametric regression models, the function $\gamma$ is estimated using some type of smoothing method, such as kernel estimation or splines. See e.g., Eubank (1999), Hastie and Tibshirani (1990), Hastie, Tibshirani, and Friedman (2001), Ruppert, Wand and Carroll (2003), and Wahba (1990) for general discussions of estimation in semiparametric regression models along with many specific results.

Here we use likelihood inference for $\beta$, eliminating $\gamma$ by treating it as a Gaussian process and averaging over it, forming a type of integrated likelihood (Berger, Liseo, and Wolpert, 1998; Severini, 2007; He and Severini, 2014). We refer to this methodology as Gaussian process semiparametric regression (GPSR).

It is important to emphasize that the Gaussian process assumption for $\gamma$ is used only as a method of eliminating it from the likelihood. In particular, we study the proposed estimators through their frequency properties in the model in which $\gamma$ is an unknown fixed function.

The GPSR approach has several advantages over more traditional methods of estimation in semiparametric models. One is that the integrated likelihood is based on observation of a Gaussian random vector, with a linear specification for the mean and a parametric covariance matrix; thus, the calculations use standard statistical software for linear models with a parametric covariance structure. Parameters appearing in the covariance function of the Gaussian process, which control the amount of smoothing, are handled automatically; a separate method to choose the "smoothing parameter" is not needed. This is particularly useful in models with correlated errors. It is well-known that choosing the smoothing parameter for semiparametric regression models is often difficult (Opsomer, Wang, and Yang, 2001). Although some progress has been made (see, e.g., De Branter et al., 2011 and Yao and Li, 2013), it is still a challenging problem in many models.

More importantly, the methodology can be applied in any model in which the integrated likelihood can be determined, making it useful in more complex models. Such a determination generally requires properties of Gaussian random functions, which are well-studied with many results available; see, for example, Ash and Gardner (1975), Laning and Battin (1956), and Rasmussen and Williams (2006). For instance, the same basic method can be used in a wide range of models, including the partially linear model (Engle, Granger, Rice and Weiss, 1986; Heckman, 1986; Speckman, 1988; Lin and Carroll, 2001), shape-invariant models 
(Lawton, Slyvestre, and Maggio, 1972; Härdle, 1990, Ch. 9), varying-coefficient models (Hastie and Tibshirani, 1993; Fan and Zhang, 1999), and models depending on the unknown function through a linear functional (Vardi and Lee, 1993); in addition, it can handle any type of parametric correlation structure for any of these models. The goal of this paper is to show that how the Gaussian process approach can be used in many semiparametric regression models by appropriately modeling the covariance structure of the data. The proposed methodology is applied to a number of examples, illustrating the usefulness of the GPSR method.

The outline of the paper is as follows. In Section 2, a review of Gaussian process regression and its application to semiparametric regression models is given. The application of the GPSR method to specific semiparametric regression models is considered in Section 3. The asymptotic properties of the estimators of the parametric components of the model are presented in Chapter 4. Section 5 considers several numerical examples. Technical details are given in the Appendix.

\section{Review of Gaussian Process Regression}

\subsection{Nonparametric regression}

Consider the model $Y_{j}=\gamma\left(z_{j}\right)+\epsilon_{j}, j=1, \ldots, n$, where $\gamma$ is an unknown function, $z_{1}, \ldots, z_{n}$ are fixed constants taking values in a set $\mathcal{Z}$, and $\epsilon_{1}, \ldots, \epsilon_{n}$ are independent normal random variables each with mean 0 and standard deviation $\sigma$. Gaussian process regression is based on modeling $\gamma$ a Gaussian process with mean function 0 and covariance function $K_{\lambda}(\cdot, \cdot)$, where, for each $\lambda, K_{\lambda}$ is a realvalued function on $\mathcal{Z} \times \mathcal{Z}$ and $\lambda \in \Lambda$ is an unknown parameter vector. In addition, $\gamma(\cdot)$ and the errors $\epsilon_{1}, \epsilon_{2}, \ldots, \epsilon_{n}$ are assumed to be uncorrelated. This distribution is often viewed as a type of prior distribution for $\gamma$.

Under the Gaussian process distribution, $\left(\gamma\left(z_{1}\right), \ldots, \gamma\left(z_{n}\right)\right)^{\mathrm{T}}$ has a multivariate normal distribution with mean vector 0 and covariance matrix based on

$$
\operatorname{Cov}\left(\gamma\left(z_{j}\right), \gamma\left(z_{k}\right)\right)=K_{\lambda}\left(z_{j}, z_{k}\right), \quad j, k=1, \ldots, n .
$$

It follows that marginal distribution of $Y \equiv\left(Y_{1}, \ldots, Y_{n}\right)^{\mathrm{T}}$ is multivariate normal with mean vector 0 and covariance matrix $\sigma^{2} I_{n}+\Sigma_{\lambda}$ where $I_{n}$ is the $n \times n$ identity matrix and $\Sigma_{\lambda}$ is the $n \times n$ matrix with $(j, k)$ th element given by (1).

For $z^{*} \in \mathcal{Z}$, the conditional expected value of $\gamma\left(z^{*}\right)$ given the data is given by $\Sigma_{\lambda}^{*}\left(\sigma^{2} I_{n}+\Sigma_{\lambda}\right)^{-1} Y$ where $\Sigma_{\lambda}^{*}$ is the $1 \times n$ matrix representing the covariance matrix of $\gamma\left(z^{*}\right)$ and $\left(\gamma\left(z_{1}\right), \ldots, \gamma\left(z_{n}\right)\right)^{\mathrm{T}}$; this may be viewed as a type of Best Linear Unbiased Predictor (BLUP). This expression, as $z^{*}$ varies, yields the 
Gaussian process regression estimator of $\gamma$, with any unknown parameters replaced by estimators. Many estimators have been proposed for these, including likelihood-based estimators as well as those based on cross-validation. See, Zhu et al. (1998), Williams (1999), Seeger (2004), Rasmussen and Williams (2006) and Murphy (2012) for general discussions of Gaussian process regression. MacKay (1999) and Sundararajan and Keerthi (2001) discuss the problem of choosing the parameters of the covariance function; Shi and Choi (2011) discuss Gaussian process regression in models for functional data.

The properties of $K_{\lambda}(\cdot, \cdot)$ are chosen to reflect the assumptions regarding the function $\gamma(\cdot)$. Suppose that $\mathcal{Z}$ is a subset of the real line. Then we generally assume that the covariance of $\gamma(z)$ and $\gamma(\tilde{z})$ is a decreasing function of $|z-\tilde{z}|$; specifically, take $K_{\lambda}(z, \tilde{z})=\tau^{2} \bar{K}(|z-\tilde{z}| / \alpha)$ where $\bar{K}$ is a decreasing, positive definite function on $[0, \infty)$ with $\bar{K}(0)=1$ and $\tau$ and $\alpha$ are positive parameters, controlling the vertical and horizontal variation of the function, respectively.

The smoothness of a Gaussian process depends on the smoothness of the function $\bar{K}$ : if $\bar{K}^{(2 m)}(0)$ exists and is finite, then the process is $m$-times mean-square differentiable (Ash and Gardiner, 1975). One choice for $\bar{K}$ is the Gaussian covariance function, $\bar{K}(t)=\exp \left(-t^{2} / 2\right), t \geq 0$. Since this function is infinitelydifferentiable, the Gaussian process is infinitely-differentiable in mean-square. For simplicity, here the covariance function of the Gaussian process is always taken to be the Gaussian covariance function. However, other covariance functions could be used without changing the basic methodology; see Abrahamsen (1997) and Rasmussen and Williams (2006, Chapter 4) for further discussion.

It is well-known that the Gaussian process approach to nonparametric regression is related to the methods based on splines. See, e.g., Ruppert, Wand, and Carroll (2003), Kimeldorf and Wahba (1970) and Wahba (1990). In the spline approach, the covariance function is chosen so that the estimate of the unknown function has the desired form.

\subsection{Gaussian process semiparametric regression}

The Gaussian process approach may also be applied to semiparametric regression models. Suppose we observe $Y_{1}, Y_{2}, \ldots, Y_{n}$ such that $Y_{j}=x_{j}^{\mathbf{T}} \beta+\gamma\left(z_{j}\right)+\epsilon_{j}$, $j=1, \ldots, n$, where $x_{1}, \ldots, x_{n}$ are $p \times 1$ vectors, $\epsilon_{1}, \ldots, \epsilon_{n}$ are unobserved independent normal random variables each with mean 0 and standard deviation $\sigma>0$, $\beta$ is an unknown parameter taking values in $\Re^{p}, z_{1}, \ldots, z_{n}$ are observed constants, taking values in a set $\mathcal{Z}$, and $\gamma$ is an unknown real-valued function on $\mathcal{Z}$.

Let $Y=\left(Y_{1}, Y_{2}, \ldots, Y_{n}\right)^{T}, X=\left(x_{1}^{T}, x_{2}^{T}, \ldots, x_{n}^{T}\right)^{T}, \epsilon=\left(\epsilon_{1}, \epsilon_{2}, \ldots, \epsilon_{n}\right)^{T}$, and $g_{\gamma}=\left(\gamma\left(z_{1}\right), \gamma\left(z_{2}\right), \ldots, \gamma\left(z_{n}\right)\right)^{T}$; then this model may be written $Y=X \beta+g_{\gamma}+\epsilon$. 
Let $\Omega_{\phi}$ denote the covariance matrix of $\epsilon$, which is assumed to have a parametric form with parameter $\phi$.

In Gaussian process semiparametric regression, we construct the integrated likelihood by taking $\{\gamma(z): z \in \mathcal{Z}\}$ to be a mean-zero Gaussian process with covariance function $K_{\lambda}(\cdot, \cdot)$, that is uncorrelated with the errors, and "integrating out" $\gamma\left(z_{1}\right), \ldots, \gamma\left(z_{n}\right)$. The result is a Gaussian likelihood with a mean depending on $\beta$ and a covariance matrix that reflects the covariance function of $\gamma$. That is, instead of including $\gamma$ in the specification of the mean of $Y$, we modify the covariance structure of $Y$ to reflect the presence of $\gamma$ in the model.

For a given choice of $K_{\lambda}(\cdot, \cdot)$, let $\Sigma_{\lambda}$ denote the $n \times n$ matrix with $(i, j)$ th element $K_{\lambda}\left(z_{i}, z_{j}\right)$. Then the integrated $\log$-likelihood function for $(\beta, \theta)$ is

$$
-\frac{1}{2} \log |V(\theta)|-\frac{1}{2}(Y-X \beta)^{\mathrm{T}} V(\theta)^{-1}(Y-X \beta) .
$$

where $V(\theta)=\Omega_{\phi}+\Sigma_{\lambda}$ and $\theta=(\phi, \lambda)$.

The covariance function of the Gaussian process is chosen to reflect the properties of the function $\gamma$. For instance, in the partially linear model, $\mathcal{Z}$ is a subset of the real line and we can take $\gamma$ to have a Gaussian covariance function. Then the integrated likelihood function is given by (2) with $V(\theta)=\sigma^{2} I_{n}+\Sigma_{\lambda}, \theta=(\sigma, \lambda)$, where $I_{n}$ denotes the $n \times n$ identity matrix.

Green, Jennison, and Seheult (1985) discuss the relationship between smoothing and covariance-based estimation in the partially linear model; see also Green (1985). Choi, Lee, and Roy (2009) consider a related approach based on an orthogonal series expansion of $\gamma$ with random coefficients.

If the model contains additional structure for $\gamma$, or a particular relationship between $\gamma$ and $\beta$, then the covariance function must be chosen to reflect these. The goal of this paper is to show how the covariance function may be chosen so that the Gaussian process approach may be applied to a wide range of semiparametric regression models, using the same basic methodology; see Section 3.

\subsection{Estimation}

For a given value of the dispersion parameter $\theta, \beta$ can be estimated by using generalized least-squares based, leading to the estimator

$$
\hat{\beta}(\theta)=\left(X^{\mathrm{T}} V(\theta)^{-1} X\right)^{-1} X^{\mathrm{T}} V(\theta)^{-1} Y .
$$

Of course, $\theta$ is generally unknown and must be estimated. 
The maximum likelihood estimator of $\theta$ can be obtained by maximizing the profile log-likelihood for $\theta$, based on the integrated likelihood, and given by

$$
\ell_{p}(\theta)=-\frac{1}{2} \log |V(\theta)|-\frac{1}{2}(Y-X \hat{\beta}(\theta))^{\mathrm{T}} V(\theta)^{-1}(Y-X \hat{\beta}(\theta)) .
$$

However, the estimators based on restricted maximum likelihood (REML) are generally considered to be preferable to the maximum likelihood estimators and that is the approach that will be used here.

The REML estimator of $\theta$ is obtained by maximizing the restricted log-likelihood $\ell^{r}(\theta) \equiv \ell_{p}(\theta)-\log \left|X^{\mathrm{T}} V(\theta)^{-1} X\right| / 2$; see, e.g., Searle, Casella, and McCulloch (1992, Chapter 6). Given the REML estimator $\hat{\theta}$ of $\theta$, an estimator of $\beta$ is given by $\hat{\beta} \equiv \hat{\beta}(\hat{\theta})$. Note that $\hat{\beta}$ may be viewed as a maximizer of a particular integrated likelihood function (He and Severini, 2014). In Section 4 , it is shown that $\hat{\beta}$ is consistent and asymptotically normal in the model in which $\gamma$ is considered to be a non-stochastic function.

The function $\gamma$ may be estimated using the Best Linear Predictor (BLP) approach used in mixed effects models; see, e.g., Robinson (1991) for a general discussion of BLPs and BLUPs and Ruppert, Wand, and Carroll (2003, Chapter 4) for a discussion of BLPs and BLUPs in linear mixed models.

Let $z^{*}$ denote an element of $\mathcal{Z}$ and consider estimation of the unknown function $\gamma$ evaluated at $z^{*}, \gamma\left(z^{*}\right)$. If the dispersion parameters are known, the BLP of $\gamma\left(z^{*}\right)$ is given by $\Sigma_{\hat{\lambda}}^{*} V(\theta)^{-1}(Y-X \hat{\beta}(\theta))$ where $\Sigma_{\lambda}^{*}$ denotes the covariance of $\gamma\left(z^{*}\right)$ and $\left(\gamma\left(z_{1}\right), \ldots, \gamma\left(z_{n}\right)\right)^{\mathrm{T}}$. Replacing $\theta$ by the REML estimator, leads to the estimated BLP of $\gamma\left(z^{*}\right), \Sigma_{\hat{\lambda}}^{*} V(\hat{\theta})^{-1}(Y-X \hat{\beta})$. The vector of "fitted values" is given by $\hat{Y}=X \hat{\beta}+\Sigma_{\hat{\lambda}} V(\hat{\theta})^{-1}(Y-X \hat{\beta})$.

The parameter $\phi$ is estimated by REML. Although the REML estimator makes an implicit adjustment for the loss of degrees of freedom due to the regression parameter of the model, $\beta$, it does not account for the loss of degrees-of-freedom associated with estimation of $\gamma$ and it is often beneficial to make a further degreesof-freedom adjustment when estimating the error variance.

Here we use an ad hoc adjustment, based on the approach described in Hastie and Tibshirani (1990), which uses properties of the "smoothing matrix". The analogue of the smoothing matrix in our context is $H=\Sigma_{\hat{\lambda}}^{*} V(\hat{\theta})^{-1}$. Therefore, we may take $h=\operatorname{tr}(H)$ as the degrees-of-freedom associated with $\gamma$ and we adjust the error variance by a factor $(n-p) /(n-p-h)$, where $p=\operatorname{dim}(\beta)$. 


\subsection{Relationship to mixed effects models}

There is a formal similarity between the GPSR methodology and methods based on a linear mixed effects (LME) model (e.g., Pinheiro and Bates, 2000) in which $\gamma\left(z_{1}\right), \gamma\left(z_{2}\right), \ldots, \gamma\left(z_{n}\right)$ are random effects. Specifically, both the integrated likelihood in the GPSR approach and the marginal likelihood in a mixed effects model are based on a distribution for the vector $g_{\gamma}$. However, there a number of important differences between the two methods.

The covariance matrix for $g_{\gamma}$ in the GPSR method is chosen to reflect the properties of $\gamma(\cdot)$ as function on $\mathcal{Z}$, while in the LME model, the covariance matrix of $g_{\gamma}$ is chosen to represent the properties of the experimental units randomly drawn from some population. Furthermore, in the LME model, the covariance structure is generally based on the structure of the data, such as in multilevel data or repeated measures; on the other hand, in the GPSR method, the covariance structure depends on the observed values of the $z_{j}$.

The most important difference between the two approaches is in the nature of the resulting inferences. In a LME model, $g_{\gamma}$ is considered to be random and the goals are to provide inferences for $\beta$ that are valid for the population from which these random effects are drawn and to provide estimates of the betweenunit variability, as reflected in the variance of the random effects. In the GPSR approach, the goal is to provide inferences for $\beta$ that are valid for an unknown, non-stochastic function $\gamma(\cdot)$; the distribution for $g_{\gamma}$ is used only as a device for eliminating $\gamma(\cdot)$ from the likelihood. In particular, parameters of the distribution of $g_{\gamma}$ have no substantive meaning for the data under consideration.

\section{Applying the GPSR Approach to Specific Semiparametric Regression Mod- els}

In order to apply the GPSR approch to a semiparametric regression model, the covariance matrix $V(\theta)$ for the integrated likelihood must be determined; the form of $V(\theta)$ will depend on the specific structure of the model. In this section, we consider this issue for several general types of semiparametric regression model.

\subsection{Partially linear models with linear constraints on the unknown function}

Consider the model $Y_{j}=X_{j} \beta+\gamma\left(z_{j}\right)+\epsilon_{j}$ where $\gamma(\cdot)$ is an unknown function and $\mathcal{Z} \subset \Re$. Suppose that $\gamma(\cdot)$ is subject to a constraint $T \gamma(\cdot)=0$ where $T$ is a known, affine function on $L_{2}(\mathcal{Z})$. Thus, we need a covariance function for $\{\gamma(z)$ : $z \in \mathcal{Z}\}$ that respects the condition $T \gamma(\cdot)=0$. To construct such a function, we may consider a mean-zero Gaussian process $\{g(z): z \in \mathcal{Z}\}$ with covariance 
function $H_{\lambda}$ of the form considered in Section 2 and then take $\{\gamma(z): z \in \mathcal{Z}\}$ to have the the conditional distribution of $g(\cdot)$ given that $T g(\cdot)=0$.

Since $\{g(z): z \in \mathcal{Z}\}$ is assumed to be a Gaussian process, this conditional distribution is identical to the distribution of

$$
g(z)-\frac{\operatorname{Cov}[g(z), T g(\cdot)]}{\operatorname{Var}(T g(\cdot))} T g(\cdot) ;
$$

see, e.g., Janson (1997, Chapter 9). It follows that $\{\gamma(z): z \in \mathcal{Z}\}$ is a mean-zero Gaussian process with covariance function

$$
K_{\lambda}(t, s)=H_{\lambda}(t, s)-\frac{\operatorname{Cov}[g(t), T g(\cdot) ; \lambda] \operatorname{Cov}[g(s), T g(\cdot) ; \lambda]}{\operatorname{Var}(T g(\cdot) ; \lambda)} .
$$

Thus, the restriction can be taken into account by simply modifying the covariance function of the process.

For two types of functions $T$ calculation of $\mathcal{K}_{\lambda}(\cdot, \cdot)$ is particularly easy. First suppose that $T g(\cdot)=g\left(z_{0}\right)-c$ where $z_{0}$ is a given element of $\mathcal{Z}$ and $c$ is a given constant. Then

$$
K_{\lambda}(t, s)=H_{\lambda}(t, s)-\frac{H_{\lambda}\left(t, z_{0}\right) H_{\lambda}\left(s, z_{0}\right)}{H_{\lambda}\left(z_{0}, z_{0}\right)} .
$$

Now suppose that

$$
\operatorname{Tg}(\cdot)=\int_{\mathcal{Z}} g(t) w(t) d t-c
$$

where $w$ is a given element of $L_{2}(\mathcal{Z})$ and $c$ is a constant. Then

$$
\operatorname{Cov}[g(s), T g(\cdot) ; \lambda]=\int_{\mathcal{Z}} H_{\lambda}(s, t) w(t) d t
$$

and

$$
\operatorname{Var}(T g(\cdot) ; \lambda)=\int_{\mathcal{Z}} \int_{\mathcal{Z}} H_{\lambda}(s, t) w(s) w(t) d s d t ;
$$

see, e.g., Ash and Gardner (1975, Chapter 1).

\subsection{Models that depend only on linear functionals of the unknown function}

Consider the model

$$
Y_{j}=X_{j} \beta+\int_{-\infty}^{\infty} H\left(z_{j}, s\right) \gamma(s) d s+\epsilon_{j}, \quad j=1, \ldots, n
$$


where $\gamma(\cdot): \Re \rightarrow \Re$ is an unknown function and $H: \Re \times \Re \rightarrow \Re$ is a known, bounded, function. Thus, the model depends on the unknown function $\gamma(\cdot)$ only through the integral

$$
\int_{-\infty}^{\infty} H(z, s) \gamma(s) d s
$$

see, e.g., Vardi and Lee (1993).

Again, we model $\gamma(\cdot)$ as a mean-zero Gaussian process with covariance function $K_{\lambda}(\cdot, \cdot)$ depending on an unknown parameter $\lambda$. Let

$$
\psi(z)=\int_{-\infty}^{\infty} H(z, s) \gamma(s) d s .
$$

Then the model may be written $Y_{j}=X_{j} \beta+\psi\left(z_{j}\right)+\epsilon_{j}$.

It may be shown (see, e.g., Laning and Battin (1956), p. 157) that the process $\{\psi(z): z \in \Re\}$ is also a mean-zero Gaussian process and that the covariance function of this process is given by

$$
Q_{\lambda}(t, s)=\int_{-\infty}^{\infty} \int_{-\infty}^{\infty} H\left(t, u_{1}\right) H\left(s, u_{2}\right) K_{\lambda}\left(u_{1}, u_{2}\right) d u_{1} d u_{2} .
$$

Thus, given $K_{\lambda}$, we can determine $Q_{\lambda}(t, s)$; also note that $H$ may depend on unknown parameters, which will then be reflected in $Q_{\lambda}$.

If $\{\gamma(z): z \in \Re\}$ is stationary and $H(t, s)=H_{0}(t-s)$ for some function $H_{0}$, then $\{\psi(z): z \in \Re\}$ is also stationary. Let $K_{\lambda}(t, s)=\bar{K}_{\lambda}(t-s)$. Then the covariance function of $\{\psi(z): z \in \Re\}$ is given by $Q_{\lambda}(t, s)=\bar{Q}_{\lambda}(t-s)$ where

$$
\bar{Q}_{\lambda}(t)=\int_{-\infty}^{\infty} \int_{-\infty}^{\infty} H_{0}\left(u_{1}\right) H_{0}\left(u_{1}+u_{2}\right) \bar{K}_{\lambda}\left(u_{2}+t\right) d u_{1} d u_{2} .
$$

\subsection{Varying-coefficient models}

A flexible approach to modeling interaction between two predictors on a response variable is a varying-coefficient model in which the coefficient of one predictor is a function of the other predictor. Specifically, consider the model $Y_{j}=\alpha+x_{j} \gamma\left(z_{j}\right)+\epsilon_{j}, j=1, \ldots, n$. See Hastie and Tibshirani (1993) for detailed discussion of varying-coefficient models.

Using the Gaussian-process approach, we take $\gamma$ to be a mean-zero Gaussian process; it follows that $x_{j} \gamma\left(z_{j}\right), j=1, \ldots, n$, are mean-zero normal random variables with correlation structure depending on the covariance function of the Gaussian process, as well as on the $x_{j}$ :

$$
\operatorname{Cov}\left(x_{j} \gamma\left(z_{j}\right), x_{k} \gamma\left(z_{k}\right)\right)=x_{j} x_{k} K_{\lambda}\left(z_{j}, z_{k}\right)
$$

where $K_{\lambda}$ is the covaraince function of the Gaussian process $\gamma(\cdot)$. 


\subsection{Shape-invariant models}

For $j=1, \ldots, n$, let $Y_{j}$ denote a response variable observed for subjects in one of two groups; let $x_{j}$ denote a group indicator variable with $x_{j}=1$ for group 1 and $x_{j}=0$ for group 2. For each $j$ let $z_{j}$ denote a fixed constant such that the mean of $Y_{j}$ given $x_{j}, z_{j}$ is of form $f_{x_{j}}\left(z_{j}\right)$ where $f_{0}, f_{1}$ are unknown functions. We assume that $f_{1}$ is a scaled and shifted version of $f_{0}: f_{1}(z)=\beta_{1}+\beta_{2} f_{0}\left(z_{j}+\beta_{3}\right)$. This is an example of a shape-invariant model, as discussed, e.g., in Härdle (1990, Ch. 9). Under this model, the mean of $Y_{j}$ is of the form $\beta_{0}+\beta_{1} x_{j}+\beta_{2}^{x_{j}} \gamma\left(z_{j}+\beta_{3} x_{j}\right)$ for an unknown function $\gamma$.

Let $\bar{K}_{\lambda}(|t-s|)$ denote the covariance of $\gamma(t)$ and $\gamma(s)$. Then the matrix $V(\theta)$ used in the GPSR approach may be based on the fact that

$$
\operatorname{Cov}\left(\beta_{2}^{x_{j}} \gamma\left(z_{j}+\beta_{3} x_{j}\right), \beta_{2}^{x_{k}} \gamma\left(z_{k}+\beta_{3} x_{k}\right)\right)=\beta_{2}^{x_{j}+x_{k}} \bar{K}_{\lambda}\left(\left|z_{j}-z_{k}+\beta_{3}\left(x_{j}-x_{k}\right)\right|\right) .
$$

\section{Asymptotic Properties of the Estimators}

\subsection{Basic approach}

The integrated likelihood in the GPSR approach corresponds to that of a multivariate normal distribution with mean $X \beta$ and covariance matrix $V(\theta)$; however, since $\gamma$ is a fixed function, the actual distribution of $Y$ is multivariate normal with mean $X \beta+g_{\gamma}$ and covariance matrix $\Omega_{\phi}$, where $g_{\gamma}=\left(\gamma\left(z_{1}\right), \ldots, \gamma\left(z_{n}\right)\right)^{\mathrm{T}}$. That is, because the likelihood used for inference is obtained by using a distribution for $\gamma(\cdot)$ but, in fact, $\gamma(\cdot)$ is a non-stochastic parameter, there is sense in which the model on which inference is based is misspecified. Hence, we must use results on the properties of likelihood-based estimators in misspecified models. Specifically, our results are based on He (2012), which considers the properties of likelihood estimators of misspecified models, extending the results of Huber (1967).

\subsection{Regularity conditions}

We assume that the following regularity conditions are satisfied.

Condition A The parameter space $\Theta$ of $\theta$ is an open subset of $\Re^{k}$. The covariance matrices $\Omega_{\phi}$ and $\Sigma_{\lambda}$ are second-order continuously differentiable in $\phi$ and $\lambda$, respectively, and are positive definite respectively for all $\phi$ and $\lambda$.

Using the spectral decomposition of a symmetric positive definite matrix, let $\Omega_{\phi}^{-\frac{1}{2}} \Sigma_{\lambda} \Omega_{\phi}^{-\frac{1}{2}}=Q \Lambda Q^{T}$, where $\Lambda=\operatorname{diag}\left(\lambda_{n 1}, \lambda_{n 2}, \ldots, \lambda_{n n}\right), \lambda_{n 1} \geq \lambda_{n 2} \geq \ldots \geq$ $\lambda_{n n}, Q=\left(q_{1}, q_{2}, \ldots, q_{n}\right)$, and $\lambda_{n i}$ and $q_{i}$ are respectively the eigenvalue and its corresponding eigenvector of the matrix $\Omega_{\phi}^{-\frac{1}{2}} \Sigma_{\lambda} \Omega_{\phi}^{-\frac{1}{2}}$ for $i=1,2, \ldots, n$. Let 
$g_{\gamma}=\left(\gamma\left(z_{1}\right), \ldots, \gamma\left(z_{n}\right)\right)^{\mathrm{T}}$ and let $\alpha=\left(\alpha_{1}, \alpha_{2}, \ldots, \alpha_{n}\right)^{T}$ denote the coordinates of $\Omega_{\phi}^{-\frac{1}{2}} g_{\gamma}$ with respect to the eigenvectors $q_{1}, q_{2}, \ldots, q_{n}$, that is, $\alpha=Q^{T} \Omega_{\phi}^{-\frac{1}{2}} g_{\gamma}$.

Condition B For any $\theta \in \Theta$,

$$
\begin{aligned}
& \sum_{j=1}^{n} \frac{\left|\alpha_{j}\right|}{1+\lambda_{n j}}<C_{1} \text { for all } n \text { for some constant } C_{1}, \\
& \left\|X^{T} \Omega_{\phi}^{-\frac{1}{2}} q_{j}\right\|<C_{2} \text { for all } j=1,2, \ldots, n, n=1,2, \ldots \text { for some constant } C_{2}, \\
& \left\|Q^{T} \Omega_{\phi}^{-\frac{1}{2}} \frac{\partial V(\theta)}{\partial \theta_{i}} \Omega_{\phi}^{-\frac{1}{2}} Q\right\|<C_{3} \text { for } i=1,2, \ldots, k \text { and some constant } C_{3} .
\end{aligned}
$$

Condition (B) is a smoothness condition on $\gamma$; it holds if $\Omega_{\phi}^{-\frac{1}{2}} g_{\gamma}$ depends most heavily on the eigenvectors of $\Omega_{\phi}^{-\frac{1}{2}} \Sigma_{\lambda} \Omega_{\phi}^{-\frac{1}{2}}$ corresponding to the large eigenvalues. As pointed out by Rasmussen and Williams (2006), for most covariance functions that are used in practice the eigenvalues of $\Sigma_{\lambda}$ are larger for its more slowly varying eigenvectors. A similar interpretation holds in the general case, after transformation by $\Omega_{\phi}^{-\frac{1}{2}}$.

Condition C The $p \times p$ matrices

$$
\frac{1}{n} X^{T} V(\theta)^{-1} X \text { and } \frac{1}{n} X^{T} V(\theta)^{-1} \frac{\partial V(\theta)}{\partial \theta_{i}} V(\theta)^{-1} X
$$

converge to positive definite matrices for any $\theta \in \Theta$ and any $i=1,2, \ldots, k$; the $p \times p$ matrix $X^{T} V(\theta)^{-1} \Omega_{\phi_{0}} V(\theta)^{-1} X / n$ converges to a $p \times p$ positive definite matrix $\tilde{\Psi}$ for any $\theta \in \Theta$; the $p \times 1$ vector $X^{T} V(\theta)^{-1} \epsilon / \sqrt{n}$ converges in distribution to a $p$-variate normal random variable $N(0, \tilde{\Psi})$ for any $\theta \in \Theta$; the $p \times 1$ vector $X^{T} V(\theta)^{-1}\left(\partial V(\theta) / \partial \theta_{i}\right) V(\theta)^{-1} \epsilon / n \stackrel{p}{\rightarrow} 0$ for any $i=1,2, \ldots, k$ and any $\theta \in \Theta$.

Condition $\mathrm{C}$ puts weak restrictions on the behavior of the sequence of covariate vectors $x_{1}, x_{2}, \ldots$ and are standard conditions in this setting.

Condition D There exists a parameter value $\theta^{*} \in \Theta$ such that

$$
\begin{aligned}
\frac{1}{n}\left[-\frac{1}{2} \operatorname{tr}\left(V\left(\theta^{*}\right)^{-1} \frac{\partial V\left(\theta^{*}\right)}{\partial \theta_{i}}\right)+\frac{1}{2} g_{\gamma}^{T}\right. & V\left(\theta^{*}\right)^{-1} \frac{\partial V\left(\theta^{*}\right)}{\partial \theta_{i}} V\left(\theta^{*}\right)^{-1} g_{\gamma} \\
& \left.+\frac{1}{2} \epsilon^{T} V\left(\theta^{*}\right)^{-1} \frac{\partial V\left(\theta^{*}\right)}{\partial \theta_{i}} V\left(\theta^{*}\right)^{-1} \epsilon\right] \stackrel{p}{\rightarrow} 0
\end{aligned}
$$


for $i=1,2, \ldots, k$ as $n$ goes to infinity; $g_{\gamma}^{T} V(\theta)^{-1}\left(\partial V(\theta) / \partial \theta_{i}\right) V(\theta)^{-1} \epsilon / n \stackrel{p}{\rightarrow} 0$ for any $i=1,2, \ldots, k$ and any $\theta \in \Theta$.

The parameter value $\theta^{*}$ plays the role of the "true value" of $\theta$ and condition $\mathrm{D}$ may be interpreted as requiring that there is a "best" value of $\theta$, in the likelihood sense; see Huber (1967). The last part of Condition D is fairly weak and generally follows from various forms of law of large numbers.

Condition E puts conditions on the asymptotic behavior of the observed Fisher information based on the restricted $\log$-likelihood $\ell^{r}$.

Condition $\mathbf{E}$ For $i, j=1,2, \ldots, k,-\ell_{\theta_{i} \theta_{j}}^{r}\left(\theta^{*}\right) / n-I_{i j}\left(\theta^{*}\right) \stackrel{p}{\rightarrow} 0$, as $n \rightarrow \infty$, where the $I_{i j}\left(\theta^{*}\right)$ is nonrandom, and $\epsilon_{i j}(\theta) \equiv-\ell_{\theta_{i} \theta_{j}}^{r}(\theta) / n-I_{i j}\left(\theta^{*}\right) \stackrel{p}{\rightarrow} 0$ when $\left|\theta-\theta^{*}\right| \rightarrow 0$ and $n \rightarrow \infty$. Furthermore, for sufficiently large $n, I$ is positive definite where $I$ is the $k \times k$ matrix whose $(i, j)^{t h}$ element is $I_{i j}\left(\theta^{*}\right)$.

\subsection{Main results}

Let $\hat{\theta}$ and $\hat{\beta}$ denote the estimators of $\theta$ and $\beta$, respectively, described in Section 2.3. Specifically, $\hat{\beta}=\hat{\beta}(\hat{\theta})$ where

$$
\hat{\beta}(\theta)=\left(X^{\mathrm{T}} V(\theta)^{-1} X\right)^{-1} X^{\mathrm{T}} V(\theta)^{-1} Y .
$$

and $\hat{\theta}$ maximizes the restricted log-likelihood function

$$
\ell^{r}(\theta) \equiv \ell_{p}(\theta)-\log \left|X^{\mathrm{T}} V(\theta)^{-1} X\right| / 2 .
$$

Theorem 4.1 establishes the consistency of $\hat{\theta}$ and gives its asymptotic distribution; the proof is given in the appendix.

Theorem 4.1 Assume that Conditions A - E hold. Then

$$
\hat{\theta}-\theta^{*} \stackrel{p}{\rightarrow} 0 \text { and } \sqrt{n}\left(\hat{\theta}-\theta^{*}\right) \stackrel{\mathcal{D}}{\rightarrow} N\left(0, I^{-1}\right) \text { as } n \rightarrow \infty
$$

where $\theta^{*}$ and $I$ are defined in conditions $\mathrm{D}$ and $\mathrm{E}$, respectively.

Theorem 4.2 establishes the consistency of $\hat{\beta}$ and gives its asymptotic distribution; the proof is given in the appendix.

Theorem 4.2 Assume that Conditions A - D hold. Then

$$
\hat{\beta} \stackrel{p}{\rightarrow} \beta_{0} \text { and } \sqrt{n}\left(\hat{\beta}-\beta_{0}\right) \stackrel{\mathcal{D}}{\rightarrow} N_{p}(0, \Psi) \text { as } n \rightarrow \infty,
$$

where $\beta_{0}$ and $\phi_{0}$ are the true values of $\beta$ and $\phi$, respectively, and

$$
\Psi=\lim _{n \rightarrow \infty}\left[\left(\frac{X^{T} V\left(\theta^{*}\right)^{-1} X}{n}\right)^{-1} \frac{X^{T} V\left(\theta^{*}\right)^{-1} \Omega_{\phi_{0}} V\left(\theta^{*}\right)^{-1} X}{n}\left(\frac{X^{T} V\left(\theta^{*}\right)^{-1} X}{n}\right)^{-1}\right] .
$$




\section{Examples}

\subsection{Example 1: Noisy evaluation of an integral}

This example is somewhat artificial but it is useful for illustrating the methods described in Sections 3.1 and 3.2. Consider the model

$$
Y_{j}=\int_{a_{j}}^{b_{j}} \gamma_{0}(t) d t+\epsilon_{j}, \quad j=1, \ldots, n
$$

where $\gamma_{0}:[0,1] \rightarrow \Re$ is an unknown smooth function, $a_{j}$ and $b_{j}$, where $0 \leq a_{j}<$ $b_{j} \leq 1$, are fixed constants for $j=1, \ldots, n$, and $\epsilon_{1}, \ldots, \epsilon_{n}$ are independent normal random variables with mean 0 and standard deviation $\sigma$. Our goal is estimation of

$$
T \gamma_{0} \equiv \int_{0}^{1} \gamma_{0}(t) d t
$$

We may write $\gamma_{0}(t)=\beta+\gamma(t), 0 \leq t \leq 1$, where $\beta$ is the unknown parameter of interest and the function $\gamma$ satisfies $T \gamma=0$. Then the model (4) may be written

$$
Y_{j}=x_{j} \beta+\int_{a_{j}}^{b_{j}} \gamma(t) d t+\epsilon_{j}, \quad j=1, \ldots, n,
$$

where $x_{j}=b_{j}-a_{j}$ and the unknown function $\gamma$ satisfies the constraint $T \gamma=0$.

Therefore, to apply the GPSR approach to estimation of $\beta$ in model (6) we must deal with two issues. The first is finding an appropriate covariance function for a Gaussian process satisfying $T \gamma=0$, which is of the form (3) in Section 3.1, with $\mathcal{Z}=[0,1], w(t)=1$, and $c=0$. The second issue is the covariance of integrals of a Gaussian process. Let

$$
W_{j}=\int_{a_{j}}^{b_{j}} \gamma(t) d t=\int_{0}^{1} I_{\left\{t \in\left(a_{j}, b_{j}\right)\right\}} \gamma(t) d t .
$$

This is of the general form considered in Section 3.2; thus,

$$
\operatorname{Cov}\left(W_{j}, W_{k}\right)=\int_{a_{k}}^{b_{k}} \int_{a_{j}}^{b_{j}} K_{\lambda}\left(t_{1}, t_{2}\right) d t_{1} d t_{2}
$$

where $K_{\lambda}$ is the covariance function of $\gamma(\cdot)$.

Combining these results, it is straightforward to show that

$$
\operatorname{Cov}\left(W_{j}, W_{k}\right)=Q\left(\left(a_{j}, b_{j}\right) ;\left(a_{k}, b_{k}\right)\right)-\frac{Q\left(\left(a_{j}, b_{j}\right) ;(0,1)\right) Q\left(\left(a_{k}, b_{k}\right) ;(0,1)\right)}{Q((0,1) ;(0,1))}
$$


where $H_{\lambda}$ is the Gaussian covariance function and $Q$ is given by

$$
Q\left(\left(a_{1}, b_{1}\right) ;\left(a_{2}, b_{2}\right)\right)=\int_{a_{2}}^{b_{2}} \int_{a_{1}}^{b_{1}} H_{\lambda}\left(t_{1}, t_{2}\right) d t_{1} d t_{2} .
$$

To investigate the properties of the estimators of $\beta$ and $\sigma$, a small simulation study was conducted. Data were simulated from the model (4) with $\gamma_{0}$ taken to be $\gamma_{0}(t)=\pi(\cos (3 \pi t)-2 \sin (4 \pi t)+\sin (\pi t / 2)) / 2$, which integrates to 1 over the interval $(0,1)$; see Figure 2.

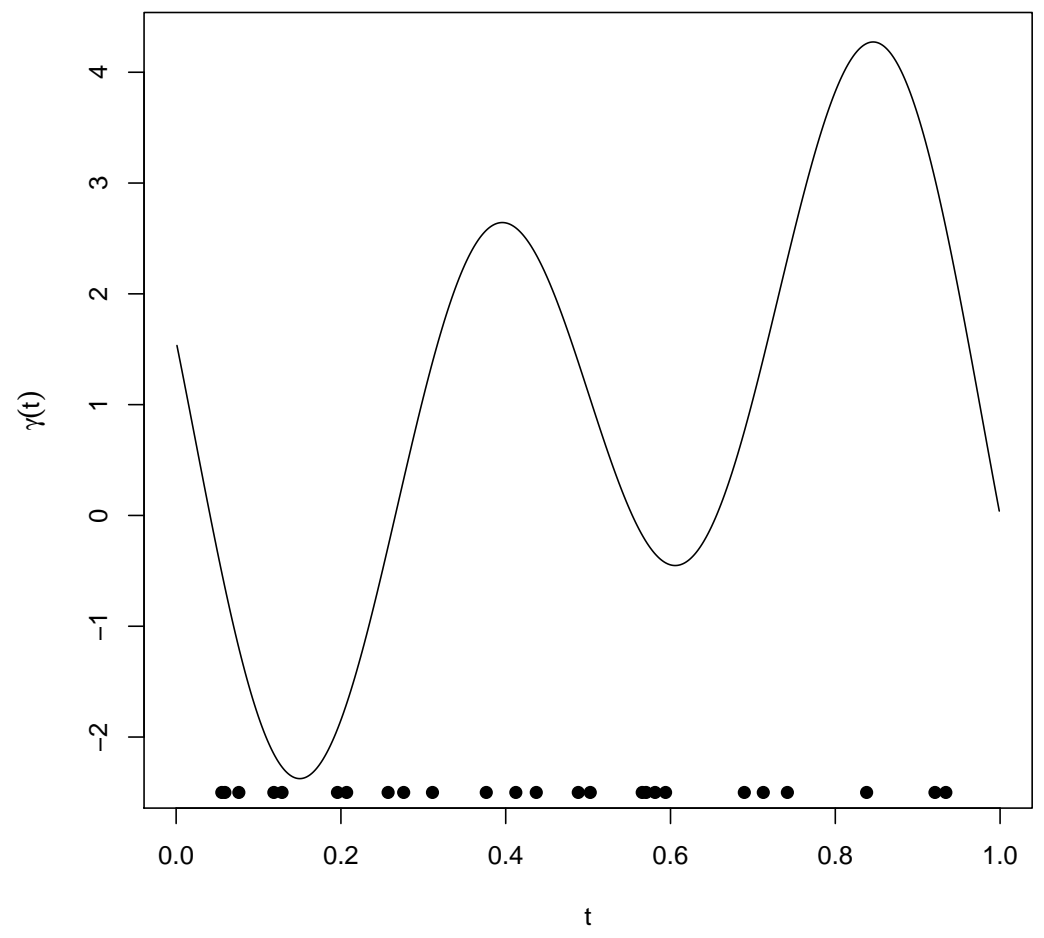

Figure 1: True Function Used in Example 1

The intervals $\left(a_{j}, b_{j}\right), j=1, \ldots, n$ were chosen by randomly choosing their midpoints uniformly $(0,1)$. The interval length was taken to be 0.2 and any interval extending outside the range $[0,1]$ was truncated. Note that the intervals were selected once and the same intervals were used in each Monte Carlo iteration; the midpoints of the intervals used are shown as dots at the bottom of the Figure 1. A sample size of $n=25$ was used and $\sigma$ was taken to be 0.2 . 
Table 1: Properties of Estimators of $\beta$ in Example 1

\begin{tabular}{cccc} 
& GPSR & Naive & CP \\
\hline Bias & -0.044 & -0.339 & -0.123 \\
SD & 0.236 & 0.216 & 0.227 \\
MSE & 0.0575 & 0.161 & 0.0666 \\
\hline
\end{tabular}

The bias, the standard deviation, and the mean-squared error of the GPSR estimator of $\beta$, are given in Table 1. The Monte Carlo sample size is 2500. For comparison, two other estimators of $\beta$ were also considered. The naive estimator is given by $\sum_{j=1}^{n} Y_{j} / \sum_{j=1}^{n} x_{j}$. The Chebychev polynomial (CP) estimator is based on an orthogonal series estimator of $\gamma_{0}$ (e.g., Härdle, 1990, Ch. 3), using Chebychev polynomials, shifted to the interval $[0,1]$ (Abramowitz and Stegun, 1964, Ch. $22)$; the number of terms in the expansion was chosen by cross-validation. Not suprisingly, the GPSR outperformed the naive estimator, which is severely biased. The CP estimator also has larger bias than does the GPSR estimator, but its standard deviation is smaller; the mean squared error (MSE) of the GPSR estimator is smaller than that of the CP estimator.

\subsection{Example 2: A shape-invariant model for clustered data}

Consider the data described in Hastie, Tibshirani, and Friedman (2001) on bone mineral density (BMD) in adolescents; these data are available in the R package "ElemStatLearn" (Halvorsen, 2012). The response variable $Y_{j}$ is the relative change in spinal BMD measurement of the subject, which is modeled as a function of age $\left(z_{j}\right)$ and gender ( $x_{j}$, with 1 for males). Preliminary analysis of the data (Hastie, Tibshirani, and Friedman, 2001, p. 128) suggests that the relationship between $Y_{j}$ and age is different for males and females, with the function relating $Y_{j}$ and age for females being a scaled and shifted version of the corresponding function for males. Under the shape-invariant model as described in Section 3.4, the mean function for males is $\beta_{0}+\beta_{1}+\beta_{2} \gamma\left(z_{j}+\beta_{3}\right)$ while the mean function for females is $\beta_{0}+\gamma\left(z_{j}\right)$. Taking $\gamma$ to be a mean-zero Gaussian process with a Gaussian covariance function,

$$
\operatorname{Cov}\left(\beta_{2}^{x_{j}} \gamma\left(z_{j}+\beta_{3} x_{j}\right), \beta_{2}^{x_{k}} \gamma\left(z_{k}+\beta_{3} x_{k}\right)\right)=\beta_{2}^{x_{j}+x_{k}} \bar{K}_{\lambda}\left(\left|z_{j}-z_{k}+\beta_{3}\left(x_{j}-x_{k}\right)\right|\right)
$$

where $\bar{K}_{\lambda}(t)=\tau^{2} \exp \left\{-t^{2} /(2 \alpha)\right\}$.

There is a further complication to this data set - some of the subjects are tested multiple times. Specifically, there are 485 observations on 261 subjects. To account for this, we modify the model to include subject-specific intercept terms 
that are taken to be normally distributed random effects with mean $\beta_{0}$ for females and mean $\beta_{0}+\beta_{1}$ for males with common variance; this induces a correlation structure between observations from the same subject.

Thus, the model includes the mean intercept $\beta_{0}$, the parameter $\beta_{1}$ representing the difference in the male and female intercepts, and six parameters that appear in the covariance structure of $Y: \beta_{2}$ and $\beta_{3}$, which describe how the functions for males and females differ, the variances of the error term and of the random intercepts, and two parameters for the Gaussian covariance function. Note that this example is different than the ones considered earlier since the parameters of primary interest, $\beta_{2}$ and $\beta_{3}$, appear in the covariance matrix of $Y$, rather than in the mean function. However, the GPSR approach is still valid and easily applied.

Figure 2 contains a plot of the data and the estimate of $\gamma$; the plotting symbol ' $x$ ' refers to males and plotting symbol ' $O$ ' refers to females. The estimates of $\beta_{2}$ and $\beta_{3}$ are 0.801 and -2.06 , respectively, with standard errors of 0.104 and 0.276 , respectively. The estimate of $\beta_{3}$ is consistent with a difference of about 2 years in the adolescent growth patterns for girls and boys.

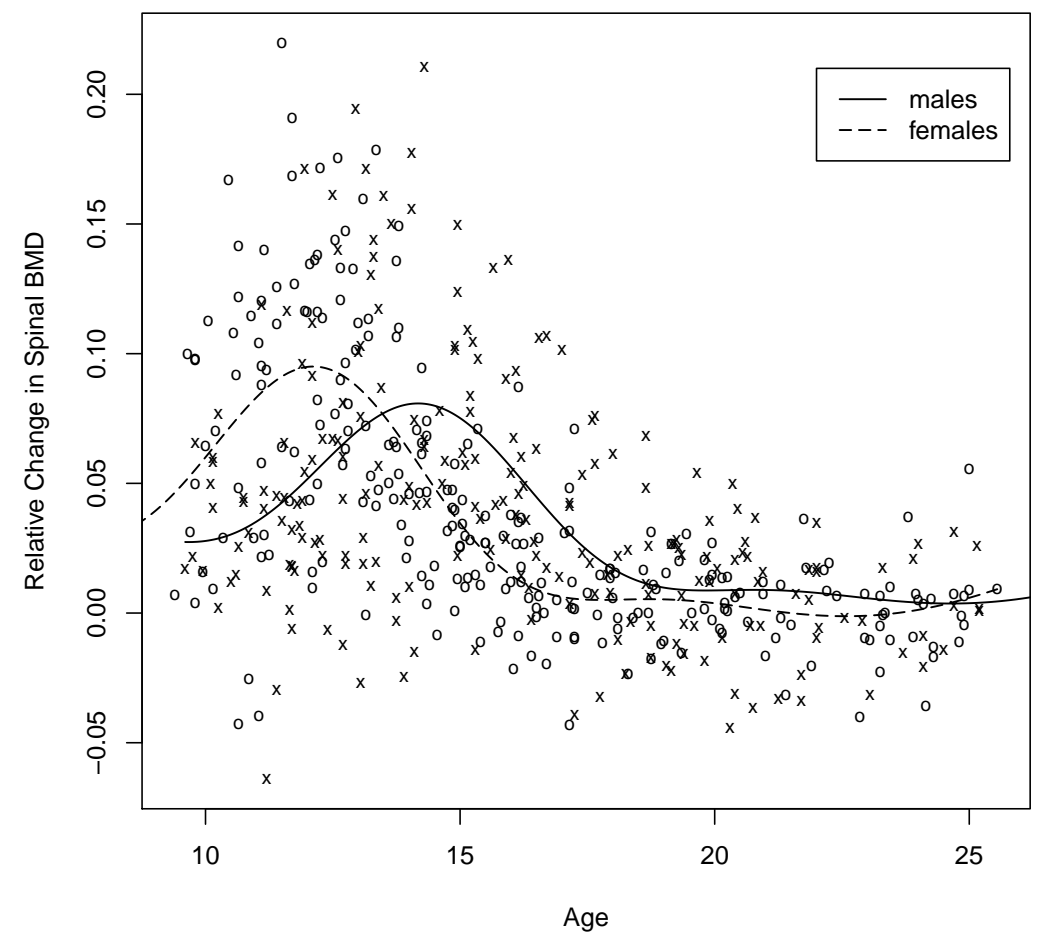

Figure 2: Function Estimates in Example 2 
An alternative approach to fitting a shape-invariant model with correlated errors is provided by the sitar package in $\mathrm{R}$ (Cole, 2015) which implements the SITAR (Super Imposition by Translation and Rotation) proposed by Cole, Donaldson, and Ben-Shlomo (2010) Under this model, the mean function for males is of the form $\delta_{i}+h\left(\exp \left(\eta_{1}+\eta_{2}\right)\left(z_{i}+\eta_{3}+\eta_{4}\right)\right)$ and the mean function for females is of the form $\delta_{i}+h\left(\exp \left(\eta_{1}\right)\left(z_{j}+\eta_{3}\right)\right)$; here $\delta_{i}$ is a subject-specific random intercept and $h$ is a natural cubic spline. The parameter $\eta_{4}$ represents the difference in timing between males and females and is analgous to the parameter $\beta_{3}$ in the GPSR approach. The parameter $\eta_{2}$ represents the difference in "velocity" of the relationship between the response and time and has an effect similar to that of $\beta_{2}$ in the GPSR, although modeled in a different way; note that

$$
\frac{\partial}{\partial z_{j}} h\left(\exp \left(\eta_{1}+\eta_{2}\right)\left(z_{j}+\eta_{3}\right)\right)=\exp \left(\eta_{1}+\eta_{2}\right) h^{\prime}\left(\exp \left(\eta_{1}+\eta_{2}\right)\left(z_{j}+\eta_{3}\right)\right)
$$

while $\partial \beta_{2} \gamma\left(z_{j}+\beta_{3}\right) / \partial z_{j}=\beta_{2} \gamma^{\prime}\left(z_{j}+\beta_{3}\right)$. See Cole, Donaldson, and Ben-Shlomo (2010) for further discussion of this model and its properties. Note that the sitar package does not allow different mean intercepts for males and females.

The parameters of the SITAR model were estimated using the bone mineral density data; the estimation procedure does not have the capacity to estimate the number of knots in the cubic spline. Thus, the number of knots was chosen to yield an effective degrees of freedom of 6 , as was done in Cole, Donaldson, and Ben-Shlomo (2010). The estimate of the timing parameter $\eta_{4}$ is -1.95 with a reported standard error of 0.456 , similar to the estimate of the timing parameter in the GPSR model (-2.06). The estimate of $\eta_{2}$ is 0.036 with a reported standard error of 0.122 ; thus, the data are consistent with $\eta_{2}=0$. The SITAR model without the parameter $\eta_{2}$ was also estimated, yielding an estimate of the timing parameter of -2.07 , with a standard error of 0.232 , in close agreement with the GPSR estimate.

To compare the GPSR and SITAR methods, a small Monte Carlo study was conducted. Data were simulated from the model described above, with same number of individuals, observations per individual, values of $z_{j}$, and so on, as in the actual dataset, using a true value of $\beta_{3}$ of -2 , a true value of $\beta_{2}$ of 1 , a true value of $\beta_{1}$ of 0 and variance parameters based on the estimates from the GPSR procedure. Thus, both the GPSR model and the SITAR model are appropriate; for the SITAR model the true value of $\eta_{2}$ is 0 and the true value of $\eta_{4}$ is -2 . For the two methods of estimation, the estimates of the timing parameters ( $\beta_{3}$ for GPSR and $\eta_{4}$ for SITAR) were computed; the results are presented in Table 2. For the sitar method, a "reduced" model without the parameter $\eta_{2}$ was also considered. The Monte Carlo sample size was 1000. 
Table 2: Properties of Estimators of the Timing Parameters in Example 2

\begin{tabular}{cccc} 
& GPSR & SITAR (full) & SITAR (reduced) \\
\hline Bias & 0.123 & 0.026 & 0.009 \\
SD & 0.325 & 0.579 & 0.303 \\
MSE & 0.121 & 0.335 & 0.092 \\
\hline
\end{tabular}

The estimator based on the full SITAR model has small bias, but a standard deviation much larger than that of the GPSR estimator; the GPSR estimator is biased, but the bias is only about one-third of a standard error. The MSE of the the GPSR estimator is considerably smaller than that of the SITAR estimator based on the full model. The SITAR estimator based on the reduced model has small bias and small standard deviation; of course, this model has two fewer parameters than does the GPSR model. In $2.2 \%$ of the simulations, the SITAR method failed to find an estimator; the GPSR succeeded in obtaining an estimator in all cases.

Although the comparison is not a direct one, the results suggest that the GPSR method is a useful alternative to the SITAR method for those cases in which the GPSR model is deemed appropriate.

\subsection{Example 3: A varying-coefficient model for clustered data}

Consider the following example. The number of goals scored by a football (soccer) team over the course of a season is roughly proportional to the number of shots on goal by that team. More skilled teams tend to have more shots and they also tend to have higher shooting percentages (the percentage of shots that result in goals) with shooting percentages ranging from around $6 \%$ for less skilled teams to $18 \%$ or higher for the best teams. This suggests a varying-coefficient model of the form $Y_{j k}=\alpha+\alpha_{j}+x_{j k} \gamma\left(z_{j k}\right)+\epsilon_{j k}$ where, for season $k$ of team $j, Y_{j k}$ denotes the goals scored per game, $x_{j k}$ denotes the shots per game, and $z_{j k}$ denotes a measure of the team's overall skill level. For this variable, we take the team's pass success percentage, the percentage of pass attempts that are successful.

Here we use data from 3 top European leagues, the English Premier League, the Spanish la Liga, and the German Bundesliga, for three seasons, 2010-11, 2011-12, 2012-13; these data are taken from www.whoscored.com. Note that, due to promotion and relegation, not all teams played in all three seasons. Because the three leagues tend to differ in the amount of scoring, we use the model $Y_{j k}=\alpha+\alpha_{j}+\beta_{L_{j}} x_{j k} \gamma\left(z_{j k}\right)+\epsilon_{j k}$, where $L_{j}$ denotes the league in which team $j$ plays, with 0 denoting the Premier League, 1 denoting La Liga, and 2 denoting the Bundesliga. Here the $\alpha_{j}$ are team-specific random effects; the parameter $\beta_{0}$ is set to 1 so that $\beta_{1}, \beta_{2}$ are interpreted relative to the value for the Premier League. 
Let $K_{\lambda}(\cdot, \cdot)$ denote the Gaussian covariance function of the Gaussian process for $\gamma$. Then Therefore, when the fixed function $\gamma$ is taken to be a Gaussian process with mean 0 and covariance function $K_{\lambda}(s, t)$, the covariance of

$$
\operatorname{Cov}\left(\beta_{L_{j}} x_{j k} \gamma\left(z_{j k}\right), \beta_{L_{m}} x_{\ell m} \gamma\left(z_{\ell m}\right)\right)=\beta_{L_{j}} \beta_{L_{m}} x_{j k} x_{\ell m} K_{\lambda}\left(z_{j k}, z_{\ell m}\right) .
$$

It follows that the covariance function of $Y$ is easily determined and is a function of $\beta_{1}$ and $\beta_{2}$, the parameters describing the differences in leagues, $\sigma^{2}$, the error variance, $\sigma_{\alpha}^{2}$, the variance of the team-specific random effect, along with the parameters appearing in the covariance function of the Gaussian process.

Using these data, the $\hat{\beta}_{1}=1.153$, with a standard error of 0.035 , and $\hat{\beta}_{2}=$ 1.229 , with a standard error of 0.043 ; the likelihood ratio statistic, based on the integrated likelihood, for testing $\beta_{1}=\beta_{2}=1$ is 21.9 . These results suggest that there are differences among the three leagues in terms of the relationship between shots on goal and scoring.

Figure 3 contains a plot of the estimated function $\hat{\gamma}$. This plot shows that the percentage of shots resulting in goals is roughly constant for teams with a pass success rate less than about $75 \%$. For teams with a pass success percentage greater than $75 \%$, the percentage of shots resulting in goals is approximately a linear function of pass success rate. Note that, for the teams and years considered here, the quartiles of pass success percentage are roughly $73 \%, 76 \%, 79 \%$, with a maximum value of $89.6 \%$ and a minimum value of $63 \%$.

\section{Discussion}

The proposed methodology gives a flexible approach to inference in a wide range of semiparametric regression models. In particular, the GPSR approach can be applied to models in which the error vector $\epsilon$ has a parametric covariance matrix $\Omega_{\phi}$, as might occur, e.g., if the errors follow a time series model or if there are random effects or random coefficients, as might be the case for clustered data or data with a hierarchical structure.

The GPSR method has several practical advantages over other methods of estimation in semiparametric regression models. As noted previously, the amount of smoothing is implicitly controlled by the parameters of the covariance function and, hence, an additional procedure for estimating the amount of smoothing is not needed. Furthermore, because the integrated likelihood is available in a closed form, computation of the estimators is straightforward and does not require specialized software. For a given value of the covariance parameter $\theta$, the 


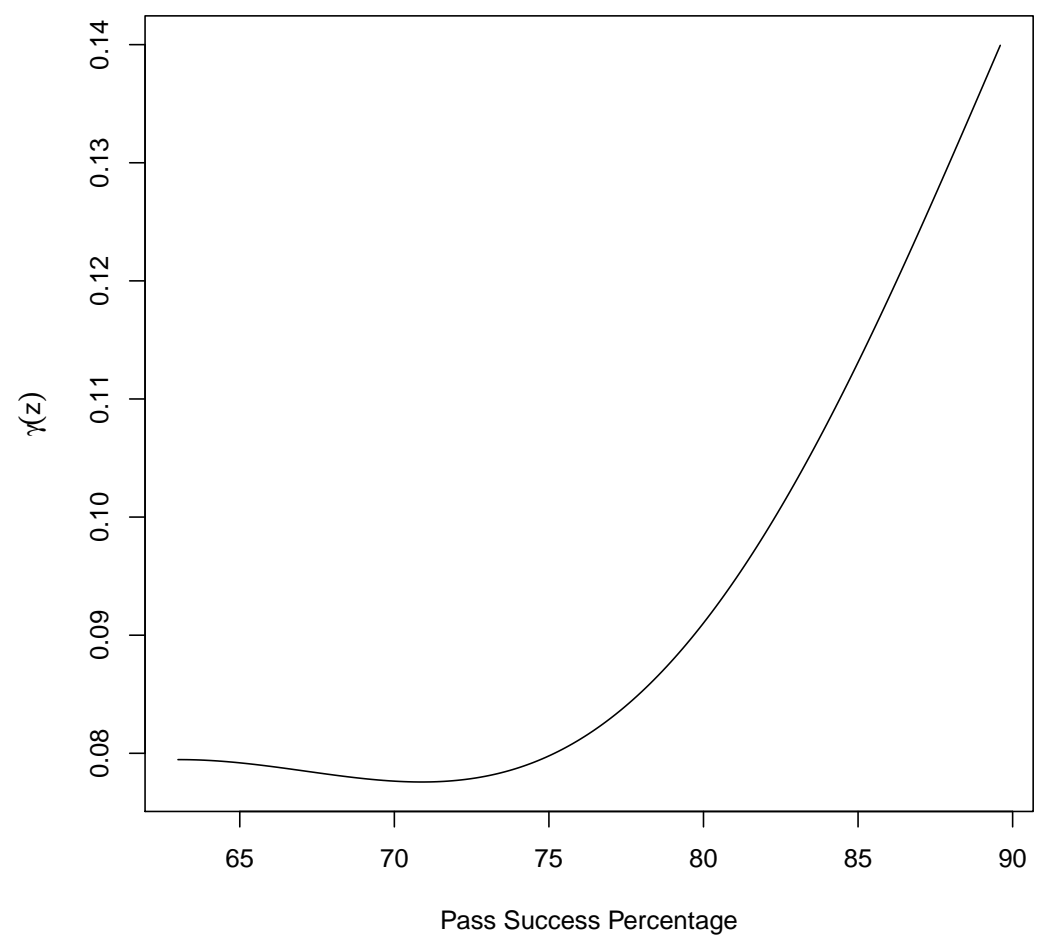

Figure 3: Estimate of $\gamma$ in Example 3

estimator of the regression parameter $\beta$ may be obtained using any method for generalized least-squares; for instance, in R (R Core Team, 2015), the function $\mathrm{lm}$. $\mathrm{gls}$ may be used. To estimate $\theta$, the restricted likelihood is used and it may be maximized using any function maximization software to obtain $\hat{\theta}$; for instance, in $\mathrm{R}$, the function opt im may be used. The estimator of $\beta$ may then be calculated using generalized least-squares with the covariance parameter taken to be $\hat{\theta}$. This approach was used in the examples discussed in Section 5.

Although the integrated likelihood for the parameter $\beta$ is based on the assumption that $\epsilon$ has a multivariate normal distribution, the method of estimation proposed here can be justified even if the normality assumption does not hold. In such cases, estimation can be based on the Gaussian likelihood method, first proposed by Whittle (1961) and used by Crowder (1985). In this method, the regression parameters, together with the dispersion parameters, are estimated using the likelihood based on assuming that $Y$ has a multivariate normal distribution; that is, the maximum likelihood estimators based on the normal-distribution assumption 
are used, even if the observations are not necessarily normally distributed. The extended generalized estimating equations (EGEE) method of Hall and Severini (1998) is equivalent to Gaussian estimation and Hall and Severini (1998) gives conditions under which this method yields consistent, asymptotically normal, estimators. Hall (2001) considers the use of REML in this context. Thus, we expect that the asymptotic properties of the estimators will continue to hold.

\section{Appendix: Technical Details}

\section{Lemma A.1}

Assume that Condition B holds. Then for $i=1,2, \ldots, k$ and any $\theta \in \Theta$

$$
X^{T} V(\theta)^{-1} g_{\gamma}=O(1) \text { and } X^{T} V(\theta)^{-1} \frac{\partial V(\theta)}{\partial \theta_{i}} V(\theta)^{-1} g_{\gamma}=O(1)
$$

By Condition $\mathrm{C}$, for any $\theta \in \Theta$ and $i=1,2, \ldots, k$, as $n \rightarrow \infty$,

$$
\begin{aligned}
& \sqrt{n}\left(X^{T} V(\theta)^{-1} X\right)^{-1} X^{T} V(\theta)^{-1} g_{\gamma} \rightarrow 0 \\
& \sqrt{n}\left(X^{T} V(\theta)^{-1} \frac{\partial V(\theta)}{\partial \theta_{i}} V(\theta)^{-1} X\right)^{-1} X^{T} V(\theta)^{-1} \frac{\partial V(\theta)}{\partial \theta_{i}} V(\theta)^{-1} g_{\gamma} \rightarrow 0 .
\end{aligned}
$$

\section{Proof:}

Note that

$$
\begin{aligned}
X^{T} V(\theta)^{-1} g_{\gamma} & =X^{T}\left(\Omega_{\phi}+\Sigma_{\lambda}\right)^{-1} g_{\gamma}=X^{T} \Omega_{\phi}^{-\frac{1}{2}}\left(I+\Omega_{\phi}^{-\frac{1}{2}} \Sigma_{\lambda} \Omega_{\phi}^{-\frac{1}{2}}\right)^{-1} \Omega_{\phi}^{-\frac{1}{2}} g_{\gamma} \\
& =X^{T} \Omega_{\phi}^{-\frac{1}{2}}\left(Q Q^{T}+Q \Lambda Q^{T}\right)^{-1} \Omega_{\phi}^{-\frac{1}{2}} g_{\gamma}=X^{T} \Omega_{\phi}^{-\frac{1}{2}} Q(I+\Lambda)^{-1} Q^{T} \Omega_{\phi}^{-\frac{1}{2}} g_{\gamma} \\
& =\sum_{i=1}^{n} \frac{\alpha_{i}}{1+\lambda_{n i}} X^{T} \Omega_{\phi}^{-\frac{1}{2}} q_{i}
\end{aligned}
$$

it follows from Condition B that

$$
\left\|X^{T} V(\theta)^{-1} g_{\gamma}\right\| \leq \sum_{i=1}^{n} \frac{\left|\alpha_{i}\right|}{1+\lambda_{n i}}\left\|X^{T} \Omega_{\phi}^{-\frac{1}{2}} q_{i}\right\|<C_{1} C_{2} .
$$

Therefore we obtain that $X^{T} V(\theta)^{-1} g_{\gamma}=O(1)$ for any $\theta \in \Theta$. 
Similarly,

$$
\begin{aligned}
& X^{T} V(\theta)^{-1} \frac{\partial V(\theta)}{\partial \theta_{i}} V(\theta)^{-1} g_{\gamma} \\
& =X^{T} \Omega_{\phi}^{-\frac{1}{2}}\left(I+\Omega_{\phi}^{-\frac{1}{2}} \Sigma_{\lambda} \Omega_{\phi}^{-\frac{1}{2}}\right)^{-1} \Omega_{\phi}^{-\frac{1}{2}} \frac{\partial V(\theta)}{\partial \theta_{i}} \Omega_{\phi}^{-\frac{1}{2}}\left(I+\Omega_{\phi}^{-\frac{1}{2}} \Sigma_{\lambda} \Omega_{\phi}^{-\frac{1}{2}}\right)^{-1} \Omega_{\phi}^{-\frac{1}{2}} g_{\gamma} \\
& =X^{T} \Omega_{\phi}^{-\frac{1}{2}} Q(I+\Lambda)^{-1} Q^{T} \Omega_{\phi}^{-\frac{1}{2}} \frac{\partial V(\theta)}{\partial \theta_{i}} \Omega_{\phi}^{-\frac{1}{2}} Q(I+\Lambda)^{-1} Q^{T} \Omega_{\phi}^{-\frac{1}{2}} g_{\gamma} .
\end{aligned}
$$

It follows from Condition B that, for some constant $C$,

$$
\left\|X^{T} V(\theta)^{-1} \frac{\partial V(\theta)}{\partial \theta_{i}} V(\theta)^{-1} g_{\gamma}\right\| \leq C C_{1} C_{2} C_{3} .
$$

The second part of the lemma follows.

\section{Proof of Theorem 4.1}

The general approach used in this proof is based on He (2012). Assumptions 2 and 3 in that paper are satisfied by choosing $K_{i}(n)$ to be $\sqrt{n}$; it remains to verify its assumption 1.

Write $\ell_{\theta_{i}}^{r}$ for the first derivative of $\ell^{r}$ with respect to $\theta_{i}$ and $V$ for $V(\theta)$. Then

$$
\begin{aligned}
\ell_{\theta_{i}}^{r}=- & \frac{1}{2} \operatorname{tr}\left(V^{-1} \frac{\partial V}{\partial \theta_{i}}\right)+\frac{1}{2}\left(g_{\gamma}^{T}+\epsilon^{T}\right) V^{-1} \frac{\partial V}{\partial \theta_{i}} V^{-1}\left(g_{\gamma}+\epsilon\right) \\
& +\frac{1}{2} \operatorname{tr}\left[\left(X^{\mathrm{T}} V^{-1} X\right)^{-1} X^{\mathrm{T}} V^{-1} \frac{\partial V}{\partial \theta_{i}} V^{-1} X\right] \\
& -\left(g_{\gamma}^{T}+\epsilon^{T}\right) V^{-1} \frac{\partial V}{\partial \theta_{i}} V^{-1} X\left(X^{T} V^{-1} X\right)^{-1} X^{T} V^{-1}\left(g_{\gamma}+\epsilon\right) \\
& +\frac{1}{2}\left(g_{\gamma}+\epsilon\right)^{T} V^{-1} X\left(X^{T} V^{-1} X\right)^{-1} X^{T} V^{-1} \frac{\partial V}{\partial \theta_{i}} V^{-1} X\left(X^{T} V^{-1} X\right)^{-1} X^{T} V^{-1}\left(g_{\gamma}+\epsilon\right) \\
\equiv I & +I I+I I I+I V+V .
\end{aligned}
$$

Consider the five terms I, II, III, IV, V defined by (A5). It follows from Condition $\mathrm{C}$ that $\left(X^{\mathrm{T}} V^{-1} X\right)^{-1} / n$ and $X^{\mathrm{T}} V^{-1}\left(\partial V / \partial \theta_{i}\right) V^{-1} X / n$ each respectively converges to a positive definite $p \times p$ matrix. Therefore, term III, divided by $n$, converges to zero for any $\theta \in \Theta$. Lemma A.1 and Condition $\mathrm{C}$ imply that

$$
\left(g_{\gamma}^{T}+\epsilon^{T}\right) V^{-1} \frac{\partial V}{\partial \theta_{i}} V^{-1} X / n \stackrel{p}{\rightarrow} 0 \text { and }\left(X^{T} V^{-1} X\right)^{-1} X^{T} V^{-1}\left(g_{\gamma}+\epsilon\right) \stackrel{p}{\rightarrow} 0 .
$$

It follows that term IV is of order $o_{p}(n)$ as $n \rightarrow \infty$ for any $\theta \in \Theta$. It similarly follows from Lemma A.1 and Condition $\mathrm{C}$ that term $\mathrm{V}$ is $o_{p}(n)$ as $n \rightarrow \infty$ for 
any $\theta \in \Theta$. Condition D implies that the sum of terms I and II evaluated at $\theta^{*}$ is $o_{p}(n)$ as $n \rightarrow \infty$. Hence, $(1 / n) \ell_{\theta_{i}}^{r}\left(\theta^{*}\right) \stackrel{p}{\rightarrow} 0$ for any $i=1,2, \ldots, k$. Therefore assumption 1 of $\mathrm{He}$ (2012) follows from Conditions $\mathrm{A}$ and $\mathrm{E}$ and this theorem follows from Theorem 2 of $\mathrm{He}$ (2012).

\section{Proof of Theorem 4.2}

It is straightforward to show that

$$
\hat{\beta}=\beta_{0}+\left(X^{T} V(\hat{\theta})^{-1} X\right)^{-1} X^{T} V(\hat{\theta})^{-1} g_{\gamma}+\left(X^{T} V(\hat{\theta})^{-1} X\right)^{-1} X^{T} V(\hat{\theta})^{-1} \epsilon .
$$

By the mean-value theorem,

$$
\begin{gathered}
\left(X^{T} V(\hat{\theta})^{-1} X\right)^{-1} X^{T} V(\hat{\theta})^{-1} g_{\gamma} \\
=\sum_{i=1}^{k}\left[\left(X^{T} V(\tilde{\theta}) X\right)^{-1}\left(X^{T} V(\tilde{\theta})^{-1} \frac{\partial V(\tilde{\theta})}{\partial \theta_{i}} V(\tilde{\theta})^{-1} X\right)\left(X^{T} V(\tilde{\theta}) X\right)^{-1} X^{T} V(\tilde{\theta})^{-1} g_{\gamma}\right. \\
\left.\quad-\left(X^{T} V(\tilde{\theta})^{-1} X\right)^{-1} X^{T} V(\tilde{\theta})^{-1} \frac{\partial V(\tilde{\theta})}{\partial \theta_{i}} V(\tilde{\theta})^{-1} g_{\gamma}\right]\left(\hat{\theta}_{i}-\theta_{i}^{*}\right) \\
+\left(X^{T} V\left(\theta^{*}\right)^{-1} X\right)^{-1} X^{T} V\left(\theta^{*}\right)^{-1} g_{\gamma}
\end{gathered}
$$

where $\tilde{\theta}=\theta^{*}+t\left(\hat{\theta}-\theta^{*}\right)$ and $t \in[0,1]$. Because $\hat{\theta} \stackrel{p}{\rightarrow} \theta^{*}$, the probability that $\tilde{\theta}$ is near $\theta^{*}$ approaches 1 ; Lemma A. 1 and Condition $\mathrm{C}$ imply that

$$
\left(X^{T} V(\theta) X\right)^{-1}\left(X^{T} V(\theta)^{-1} \frac{\partial V(\theta)}{\partial \theta_{i}} V(\theta)^{-1} X\right)\left(X^{T} V(\theta) X\right)^{-1} X^{T} V(\theta)^{-1} g_{\gamma}
$$

and

$$
\left(X^{T} V(\theta)^{-1} X\right)^{-1} X^{T} V(\theta)^{-1} \frac{\partial V(\theta)}{\partial \theta_{i}} V(\theta)^{-1} g_{\gamma}
$$

converge to zero for any $\theta$ in the close neighborhood of $\theta^{*}$, so both of them evaluated at $\tilde{\theta}$ are bounded with probability approaching 1 . Hence, by the convergence in probability of $\hat{\theta}$ to $\theta^{*}$ and Lemma A. 1, we have that $\left(X^{T} V(\hat{\theta})^{-1} X\right)^{-1} X^{T} V(\hat{\theta})^{-1} g_{\gamma}$ converges to 0 in probability as $n \rightarrow \infty$.

Using an argument similar to the one used with equation (A6),

$$
\left(X^{T} V(\hat{\theta})^{-1} X\right)^{-1} X^{T} V(\hat{\theta})^{-1} \epsilon-\left(X^{T} V\left(\theta^{*}\right)^{-1} X\right)^{-1} X^{T} V\left(\theta^{*}\right)^{-1} \epsilon \stackrel{p}{\rightarrow} 0,
$$

and, hence, that $\left(X^{T} V(\hat{\theta})^{-1} X\right)^{-1} X^{T} V(\hat{\theta})^{-1} \epsilon \stackrel{p}{\rightarrow} 0$ as $n \rightarrow \infty$. Therefore it follows from condition $\mathrm{C}$ that $\hat{\beta} \stackrel{p}{\rightarrow} \beta_{0}$ as $n$ goes to infinity. 
It is straightforward to show that

$\sqrt{n}\left(\hat{\beta}-\beta_{0}\right)=\sqrt{n}\left(X^{T} V(\hat{\theta})^{-1} X\right)^{-1} X^{T} V(\hat{\theta})^{-1} g_{\gamma}+\left(X^{T} V(\hat{\theta})^{-1} X\right)^{-1} \sqrt{n} X^{T} V(\hat{\theta})^{-1} \epsilon$

so that using a argument similar to the one used with equation (A6),

$$
\sqrt{n}\left(X^{T} V(\hat{\theta})^{-1} X\right)^{-1} X^{T} V(\hat{\theta})^{-1} g_{\gamma}-\sqrt{n}\left(X^{T} V\left(\theta_{n}^{*}\right)^{-1} X\right)^{-1} X^{T} V\left(\theta_{n}^{*}\right)^{-1} g_{\gamma} \stackrel{p}{\rightarrow} 0
$$

so by Lemma A.1 and condition $\mathrm{C}$ we obtain that

$$
\sqrt{n}\left(X^{T} V(\hat{\theta})^{-1} X\right)^{-1} X^{T} V(\hat{\theta})^{-1} g_{\gamma} \stackrel{p}{\rightarrow} 0 \quad \text { as } \quad n \rightarrow \infty .
$$

Finally, again using an argument similar to the one used with equation (A6),

$$
\left(X^{T} V(\hat{\theta})^{-1} X\right)^{-1} \sqrt{n} X^{T} V(\hat{\theta})^{-1} \epsilon \text { and }\left(X^{T} V\left(\theta^{*}\right)^{-1} X\right)^{-1} \sqrt{n} X^{T} V\left(\theta^{*}\right)^{-1} \epsilon
$$

converge in distribution to the same random variable; it follows from Condition $\mathrm{C}$ that $\left(X^{T} V(\hat{\theta})^{-1} X\right)^{-1} \sqrt{n} X^{T} V(\hat{\theta})^{-1} \epsilon \stackrel{D}{\rightarrow} N(0, \Psi)$. The result follows.

\section{Acknowledgement}

The work of TAS was supported by NSF grant DMS-1308009.

\section{REFERENCES}

Abrahamsen, P. (1997). A Review of Gaussian Random Fields and Correlation Functions, 2nd ed. Oslo: Norwegian Computing Center.

Abramowitz, M. and Stegun, I. A. (1964). Handbook of Mathematical Functions with Formulas, Graphs, and Mathematical Tables. New York: Dover.

Ash, R. B. and Gardner, M. F. (1975). Topics in Stochastic Processes. New York: Academic Press.

Berger, J. O., Liseo, B., and Wolpert, R. (1998). Integrated likelihood functions for eliminating nuisance parameters (with discussion). Statist. Sci., 14, 1-28.

Choi, T., Lee. J., and Roy. A. (2009). A note on the Bayes factor in a semiparametric regression model. J. Mult. Anal. 100, 1316-1327.

Cole, T. (2015). sitar: Super Imposition by Translation and Rotation Growth Curve Analysis.

http://CRAN.R-project.org/package=sitar. 
Cole, T. J., Donaldson, M. D., and Ben-Schlomo, Y. (2010). SITAR - a useful instrument for growth curve analysis. Int. J. Epidemiol. 39, 1558-1566.

Crowder, M. (1985). Gaussian estimation for correlated binomial data. $J . R$. Statist. Soc. B 47, 229-237.

De Branter, K., De Branter J., Suykens, J. A. K., and De Moor, B. (2011). Kernel regression in the presence of correlated errors. J. Machine Learning 12, 19551976.

Engle, R., Granger, W., Rice, J. and Weiss, A. (1986). Semiparametric estimates of the relation between weather and electricity sales. J. Amer. Statist. Assoc. 81, 310-320.

Eubank, R. L. (1999). Nonparametric Regression and Spline Smoothing. New York: Marcel Dekker.

Fan, J. and Zhang, W. (1999). Statistical estimation in varying coefficient models. Ann. Statist. 27, 1491-1518.

Green, P. J. (1985). Linear models for field trials, smoothing and cross-validation. Biometrika 72, 527-37.

Green, P., Jennision, C. and Seheult, A. (1985). Analysis of field experiments by least squares smoothing. J. R. Statist. Soc. B 47, 299-315.

Hall, D. B. (2001). On the application of extended quasi-likelihood to the clustered data case. Can. J. Statist. 29, 77-97.

Hall, D. B. and Severini, T. A. (1998). Extended generalized estimating equations for clustered data. J. Amer. Statist. Assoc. 93, 1365-1375.

Halvorsen, K. (2012). ElemstatLearn. http://CRAN.R-project.org/package=ElemStatLearn.

Härdle, W. (1990). Applied Nonparametric Regression. Cambridge: Cambridge University Press.

Hastie T. J. and Tibshirani, R. J. (1990). Generalized Additive Models. London: Chapman and Hall.

Hastie, T. and Tibshirani, R. (1993). Varying-coefficient Models. J. R. Statist. Soc. B 55, 757-796.

Hastie, T., Tibshirani, R. and Friedman, J. (2001). The Elements of Statistical Learning: Data Mining, Inference, and Prediction. New York: Springer.

He, H. (2012). The Properties of maximum pseudo-likelihood estimators of mis- 
specified models in general cases. Unpublished.

He, H. and Severini, T. A. (2014). Integrated likelihood inference in semiparametric regression models. Metron 72, 185-199.

Heckman, N.E. (1986). Spline smoothing in a partly linear model. J. R. Statist. Soc. B 48, 244-248.

Huber, P. J. (1967). The behavior of maximum likelihood estimates under nonstandard conditions. Proc. Fifth Berkeley Symp. on Math. Statist. Prob., Vol 1, pp. 221-233, Berkeley: University of California Press

Kimeldorf, G. S. and Wahba, G. (1970). A correspondence between Bayesian estimation on stochastic processes and smoothing by splines. Ann. Math. Statist.41. 495-502.

Laning, J. H. and Battin, R. H. (1956). Random Process in Automatic Control. New York: McGraw-Hill.

Lawton, W. H., Sylvestre, E. A., and Maggio, M. S. (1972). Self modeling nonlinear regression. Technometrics 14, 513-532.

Lin, X. and Carroll, R. J. (2001). Semiparametric regression for clustered data. Biometrika 88, 1179-1185.

MacKay, D. J. C. (1999). Comparison of approximate methods for handling hyperparameters. Neural Comp. 11, 1035-1068.

Murphy, K. P. (2012). Machine Learning: A Probabilistic Perspective. Cambridge: MIT Press.

Opsomer, J., Wang, Y., and Yang, Y. (2001). Nonparametric regression with correlated errors. Statist. Sci 16, 134-153.

Pinheiro, J. and Bates, D. (2000). Mixed-Effects Models in S and S-PLUS. New York: Springer.

R Core Team (2015). R: A language and environment for statistical computing. R Foundation for Statistical Computing, Vienna, Austria. URL http://www.Rproject.org/.

Rasmussen, C. E. and Williams, C. K. I. (2006). Gaussian Processes for Machine Learning. Cambridge: The MIT Press.

Robinson, G. K. (1991). That BLUP is a good thing: the estimation of random effects (with discussion). Statist. Sci. 6, 15-51.

Ruppert, D., Wand, M. P., and Carroll, R. J. (2003). Semiparametric Regression. 
Cambridge: Cambridge University Press.

Searle, S. R., Casella, G. and McCulloch, C. E. (1992). Variance Components. New York: Wiley.

Seeger, M. (2004). Gaussian processes for machine learning. International Journal of Neural Systems, 14, 69-106.

Severini, T. A. (2007). Integrated likelihood functions for non-Bayesian inference. Biometrika 94, 529-542.

Shi, J. Q. and Choi, T. (2011). Gaussian Process Regression Analysis for Functional Data. New York: Chapman and Hall.

Speckman, P. E. (1988). Kernel smoothing in partial linear models. J. R. Statist. Soc. B 50, 413-436.

Sundararajan, S. and Keerthi, S. S. (2001). Predictive approaches for choosing hyperparameters in Gaussian processes. Neural Comp. 13, 1103-1118.

Vardi, Y. and Lee, D. (1993). From image deblurring to optimal investments: maximum likelihood solutions for positive linear inverse problems (with discussion). J. R. Statist. Soc. B 55, 569-612.

Wahba, G. (1990). Spline Models for Observational Data. Philadelphia: SIAM.

Whittle, P. (1961). Gaussian estimation in stationary time series. Bull. Int. Statist. Inst. 39, 1-26.

Williams, C. K. I. (1999). Prediction with Gaussian processes: From linear regression to linear prediction and beyond. In Learning in Graphical Models, M. I. Jordan (ed.) , pp. 599-621. Cambridge: The MIT Press.

Yao, W. and Li, R. (2013). New local estimation procedure for a non-parametric regression function for longitudinal data. J. R. Statist. Soc. B 75, 123-138.

Zhu, H., Williams, C. K. I., Rohwer, R. J., and Morciniec, M. (1998). Gaussian regression and optimal finite-dimensional linear models. In Neural Networks and Machine Learning, C. M. Bishop (ed.)., pp. 315-332. Berlin:SpringerVerlag. 